\title{
Cisto da bifurcação vestibular: Relato de caso clínico
}

\author{
Vestibular bifurcation cyst: Clinical case report \\ Quiste de bifurcación vestibular: Reporte de caso clínico
}

\section{Resumo}

O cisto da bifurcação vestibular é um cisto odontogênico inflamatório incomum que se desenvolve na face vestibular do primeiro molar inferior permanente, em crianças de 5 a 13 anos. Clinicamente apresenta sensibilidade, edema, bolsa periodontal e secreção com gosto desagradável. Radiograficamente, se mostra como uma lesão radiolúcida unilocular e bem delimitada envolvendo a bifurcação vestibular e a região da raiz do dente em questão. Os ápices radiculares dos molares estão, caracteristicamente, voltados para a cortical lingual mandibular. Este estudo teve como objetivo relatar um caso clínico de uma criança com cisto da bifurcação vestibular. Trata-se de um paciente do gênero masculino, 7 anos de idade, apresentando edema na região da mandíbula direita, dor a palpação, presença de abscesso periodontal com secreção purulenta na região do dente 85 e 46. Na tomografia computadorizada de feixe cônico (TCFC) mostrou imagem radiolúcida bem delimitada, expansiva e unilocular envolvendo a coroa e a bifurcação vestibular do elemento 46, rompendo a cortical óssea alveolar vestibular, compatível com Cisto da Bifurcação Vestibular. Concluiu-se que o cisto da bifurcação vestibular é uma doença pediátrica que ocorre na área de bifurcação da face vestibular de um primeiro molar inferior permanente em erupção e aparece principalmente na primeira década de vida.

Palavras-chave: Tomografia computadorizada de feixe cônico; Cistos odontogênicos; Patologia bucal.

\begin{abstract}
The vestibular bifurcation cyst is an uncommon inflammatory odontogenic cyst that develops on the vestibular face of the permanent lower first molar in children aged 5 to 13 years. Clinically, it presents sensitivity, edema, periodontal pocket and secretion with an unpleasant taste. Radiographically, it is shown as a unilocular and well-defined radiolucent lesion involving the vestibular bifurcation and the root region of the tooth in question. The root apices of the molars are characteristically facing the mandibular lingual cortex. This study aimed to report a clinical case of a child with a vestibular bifurcation cyst. It is a male patient, 7 years old, presenting edema in the region of the right jaw, pain on palpation, presence of periodontal abscess with purulent secretion in the region of tooth 85 and 46. In the CFFC he showed a well-defined radiolucent image, expansive and unilocular involving the crown and vestibular bifurcation of element 46, disrupting the vestibular alveolar bone cortex, compatible with Vestibular Bifurcation Cyst. It was concluded that the vestibular bifurcation cyst is a pediatric disease that occurs in the bifurcation area of the buccal surface of an erupting permanent lower first molar and appears mainly in the first decade of life.
\end{abstract}

Keywords: Cone-beam computed tomography; Odontogenic cyst; Pathology oral.

\section{Resumen}

El quiste de bifurcación vestibular es un quiste odontogénico inflamatorio poco común que se desarrolla en la cara vestibular del primer molar inferior permanente en niños de 5 a 13 años. Clínicamente presenta sensibilidad, edema, 
bolsa periodontal y secreción de sabor desagradable. Radiográficamente, se muestra como una lesión radiolúcida unilocular bien definida que involucra la bifurcación vestibular y la región radicular del diente en cuestión. Los ápices de la raíz de los molares se encuentran de manera característica frente a la corteza lingual mandibular. Este estudio tuvo como objetivo reportar un caso clínico de un niño con un quiste de bifurcación vestibular. Se trata de un paciente masculino, de 7 años, que presenta edema en la región de la mandíbula derecha, dolor a la palpación, presencia de absceso periodontal con secreción purulenta en la región del diente 85 y 46. En el CFFC mostró un radiolúcido bien definido. imagen, expansiva y unilocular que involucra la corona y bifurcación vestibular del elemento 46, con ruptura de la corteza ósea alveolar vestibular, compatible con Quiste de Bifurcación Vestibular. Se concluyó que el quiste de bifurcación vestibular es una enfermedad pediátrica que se presenta en el área de bifurcación de la superficie bucal de un primer molar inferior permanente en erupción y aparece principalmente en la primera década de la vida.

Palabras clave: Tomografia computarizada de haz cónico; Quiste odontogênico; Patología bucal.

\section{Introdução}

Os cistos odontogênicos são resultantes da proliferação de remanescentes epiteliais originados na hora da formação do elemento dentário. Isso pode ocorrer tanto na maxila quanto na mandíbula, locais em que infecções e traumas são relativamente comuns. Devido a essas duas causas a resposta inflamatória é ativada, dando início a formação do cisto odontogênico inflamatório (Lino et al., 2017).

Nota-se na literatura, que existe uma porcentagem pouco significante para os relatos de casos do cisto da bifurcação vestibular (CBV): 3,0\% de todos os cistos mandibulares e 3,7 de cistos odontogênicos (Ramos et al., 2012).

É válido ressaltar que o CBV é geralmente confundido com outras entidades patológicas devido as suas semelhanças clínicas e radiográficas e, também, devido às variantes nas suas nomenclaturas. O cisto paradental (CP), um de seus diagnósticos diferenciais, possui localização na região dos terceiros molares inferiores semi-inclusos. Já o CBV em primeiros e segundos molares inferiores (Bautista et al., 2019).

Encontrado em crianças com a faixa etária de 5 a 13 anos, sem apresentar predileção por sexo, o CBV se apresenta com uma característica clínica de abaulamento ósseo na região do dente afetado. Tal característica é de extrema importância para alertar os responsáveis, a fim de uma procura imediata pelo cirurgião-dentista (Friedrich, Scheuer, \& Zustin, 2014).

Clinicamente, observa-se aumento da profundidade de sondagem com bolsa periodontal na face vestibular, além de dor, aumento de volume, abscesso localizado e outras características radiográficas (Kim et al., 2018).

O cisto da bifurcação vestibular não é encontrado na maxila e não há relato de casos em adultos, visto que o mesmo ocorre na época de formação do germe dentário ou um pouco antes da erupção do dente devido ao estímulo dos restos epiteliais (Grauwe et al., 2018).

É notório que as lesões císticas podem ser tratadas das seguintes formas: tratamento endodôntico (conservador) ou cirúrgico. O cirúrgico pode ser dividido em enucleação e marsupialização (Ramos et al. 2012). A enucleação cística consiste em uma técnica de remoção total da cavidade patológica sem dissecção, ou seja, sem sua ruptura. Tal manobra só é possível devido ao tecido conjuntivo fibroso entre o componente epitelial e a parede óssea, formando, assim, um plano de clivagem que separa cisto da cavidade. É necessária, a abordagem cirúrgica atenciosa para não deixar restos císticos dentro do sítio contaminado, podendo, posteriormente, acontecer a reinfecção e recidiva do mesmo, a qual pode ocorrer variando de meses a anos (Ellis III, 2015; Faria et al., 2013).

O objetivo deste trabalho foi relatar um caso clínico de uma criança com cisto da bifurcação vestibular, descrevendo suas características clínicas, radiográficas e histológicas, assim como possíveis diagnósticos diferenciais e tratamento. 


\section{Metodologia}

Este estudo, trata-se de um relato de caso, caracterizado como um estudo descritivo, exploratório e qualitativo (Pereira et al., 2018).

Este estudo foi submetido ao Comitê de Ética em Pesquisa do Centro Universitário de Volta Redonda (CoEPs) UniFOA, com número CAAE 34481720.6.0000.5237 e aprovado em 12/07/2020. A publicação deste estudo foi autorizada pelos responsáveis pela criança por meio da assinatura de um Termo de Consentimento Livre e Esclarecido e de uso de imagem.

\section{Relato de Caso}

Paciente gênero masculino, 7 anos, procedente do Município de Volta Redonda/RJ compareceu acompanhado dos pais ao médico otorrinolaringologista em julho de 2019. A queixa principal era aumento de volume em 1/3 terço inferior direito de face, dor aguda na região do elemento 46. Diante dos sinais e sintomas foi prescrito o Cetoprofeno e Amoxicilina + Clavulanato. Porém, quatro dias após o uso das medicações, o processo evoluiu com aumento de volume e hiperemia local e o paciente retornou ao otorrinolaringologista que o encaminhou ao Buco-Maxilo-Facial (BMF) para avaliação.

$\mathrm{Na}$ consulta com o BMF, feita no mesmo dia, foi realizado a anamnese e exame físico. Na anamnese, foi relatado pela mãe, a realização da cirurgia de postectomia sob anestesia geral e sem intercorrências. Nenhum relato de alergias ou antecedentes mórbidos individuais.

No exame físico intra-oral, observou-se que o paciente apresentava boa higiene oral e ausência de lesão cariosa. Entretanto, detectou-se presença de sinais clínicos de abscesso periodontal na região do dente segundo molar decíduo inferior direito (85), com tumefação e secreção purulenta na gengiva marginal do elemento, além de sintomatologia dolorosa à palpação. Além disso, foi avaliado o lado oposto que se encontrava dentro dos padrões de normalidade. Na avaliação extra-oral foi observado discreto edema na região da mandíbula direita. O paciente não apresentava restrição da abertura de boca, febre, mal-estar geral e sua dieta estava normal. Foi solicitado um exame de radiografia panorâmica que mostrou presença de imagem radiolúcida difusa associada à raiz distal e região de furca do elemento primeiro molar permanente inferior direito (46) em formação (rizogênese incompleta) (Figura 1). O elemento 85 radiograficamente encontra-se dentro dos padrões de normalidades.

Figura 1. Radiografia panorâmica mostrando presença de imagem radiolúcida associada à raiz distal e região de furca do elemento 46 .

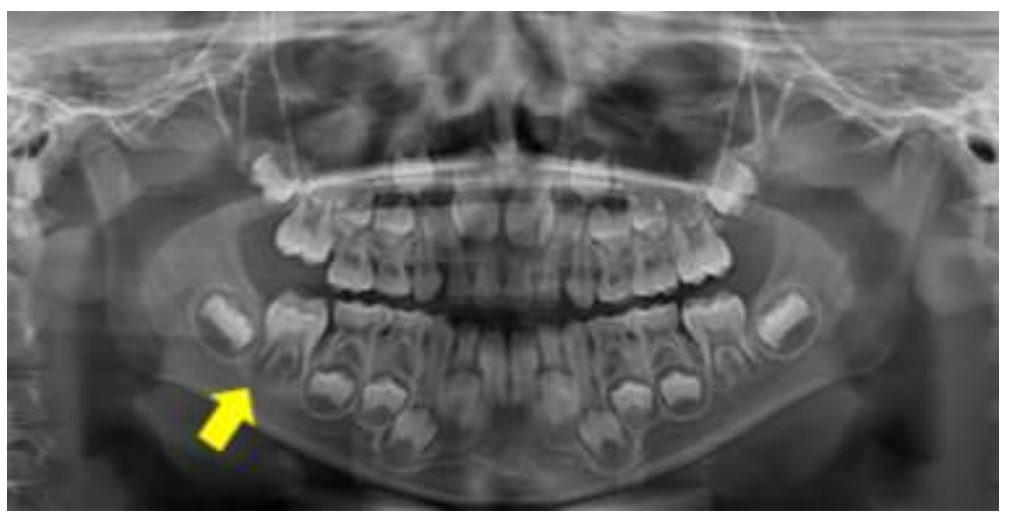

Fonte: Autores.

Logo, optou-se pela continuação do uso da Amoxicilina e Clavulanato e suspensão do Cetoprofeno. Foram adicionados alguns cuidados locais como o bochecho com água morna $6 \mathrm{x}$ ao dia, bochecho com clorexidina $0,12 \%$ aquosa de 
12/12hrs (2x por dia), durante 1 minuto (após boa higienização oral) e ainda, foi solicitado exames complementares de hemograma completo e glicemia.

Na consulta seguinte, foi analisado o hemograma completo e glicemia que se encontravam dentro dos padrões de normalidade. Houve melhora de $90 \%$ do edema facial. Foi, então, realizada coleta de material da região vestibular do elemento 85 para cultura e antibiograma que obteve resultado negativo. Mantidos os cuidados locais orientados no dia.

Retornou na próxima consulta sem sintomas, sem edema facial e abertura de boca normal. Houve regressão da fístula na região do 85/46. No entanto, na palpação local se observa abaulamento ósseo e consistente (expansão da cortical).

Diante das características clínicas e radiográficas, foi solicitado exame de tomografia computadorizada de feixe cônico (TCFC) para melhor avaliação das características da entidade patológica. Esta mostrou presença de imagem hipodensa (radiolúcida) bem delimitada, expansiva e unilocular envolvendo a coroa e a bifurcação vestibular do elemento 46, rompendo a cortical óssea alveolar vestibular. Notou-se presença de imagem compatível com periostite proliferativa da cortical alveolar vestibular sobrejacente (osso reacional). O elemento 46 estava em formação (rizogênese incompleta) e em processo de erupção. Os ápices se apresentavam voltados para a cortical lingual (Figuras 2A; 2B). A hipótese diagnóstica foi de Cisto da Bifurcação Vestibular e seu diagnóstico diferencial foi o Cisto Dentígero. Para confirmação do radiodiagnóstico foi sugerido exame histopatológico.

Figura 2. Exame de TCFC. (A) Reconstrução panorâmica mostrando o elemento 46 em erupção com imagem hipodensa envolvendo a coroa e a bifurcação vestibular do elemento 46. (B) Reconstrução 3D ilustrando rompimento de cortical óssea vestibular. (C) Cortes transaxiais e (E) Cortes Axiais evidenciando a expansão e rompimento da cortical óssea alveolar vestibular e, também, o posicionamento dos apices do elemento 46 voltados para a cortical óssea alveolar lingual. (C) Cortes transaxiais, (D) Cortes Sagitais e (E) Cortes Axiais sugerindo presença de imagem compatível com periostite proliferativa da cortical alveolar vestibular sobrejacente ao elemento 46 (osso reacional).

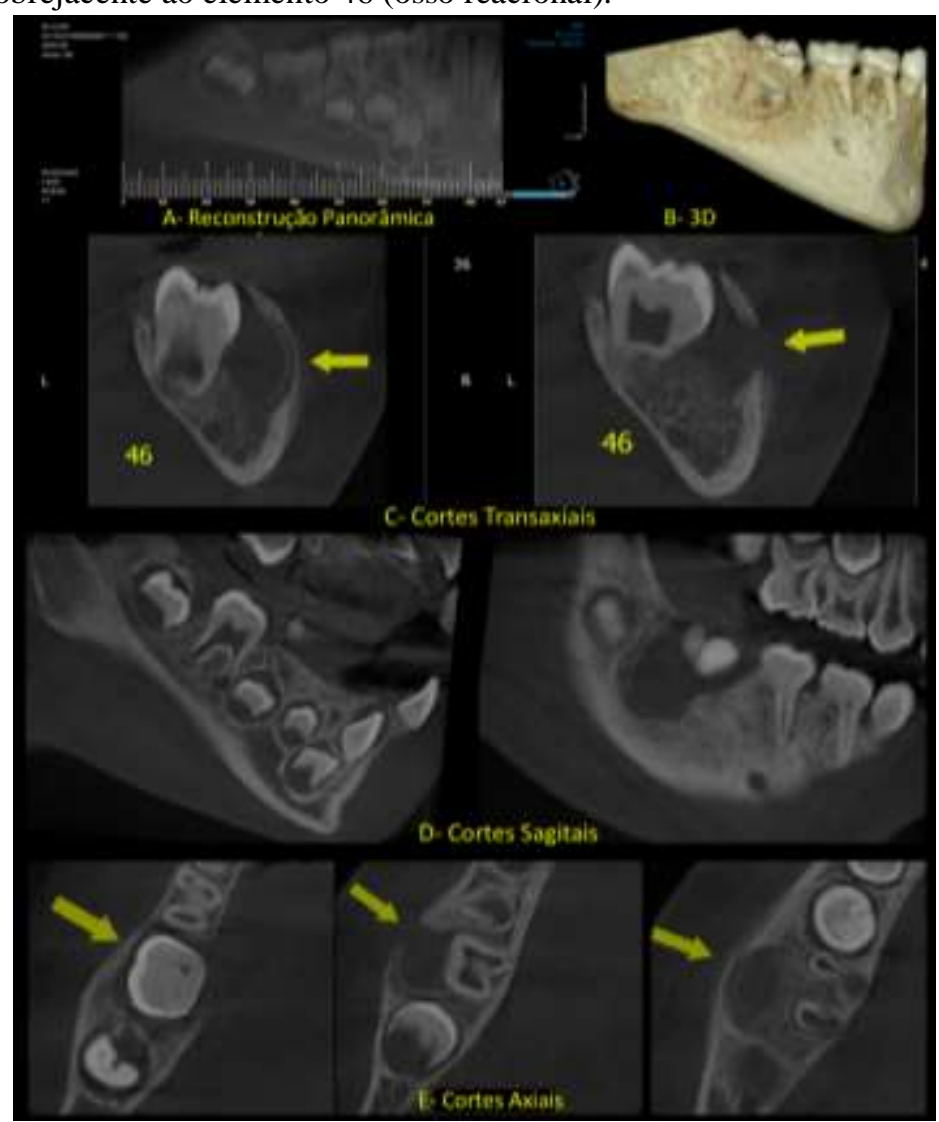

Fonte: Autores. 
Após avaliação das imagens tomográficas, decidiu-se pela cirurgia por enucleação do cisto sob anestesia geral em ambiente hospitalar devido à pouca idade do paciente. Foi solicitado avaliação pré-operatória para anestesia geral (exame de sangue, urina, Radiografia de tórax, ECG e risco cirúrgico).

O paciente foi operado sob anestesia geral, em julho de 2019, sendo realizada enucleação do cisto com reparação óssea e tecidual com fibrina leucoplaquetária autóloga sem extração do elemento dentário envolvido. Seus responsáveis foram orientados sobre os cuidados locais pós-operatório (Figura 3).

Figura 3. Enucleação cística.

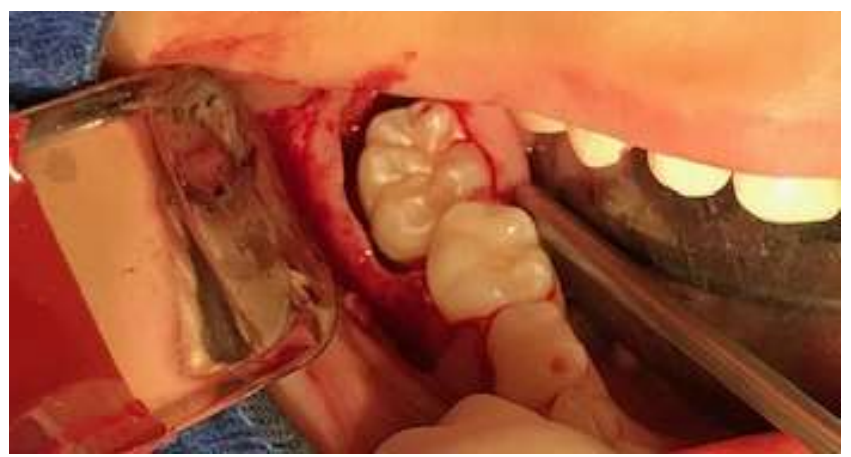

Fonte: Autores.

O paciente foi submetido a biópsia incisional; o espécime foi removido e armazenado em formaldeído $10 \%$, enviado para exame histopatológico e confirmado o diagnóstico de Cisto da Bifurcação Vestibular (Figura 4).

Figura 4. A: Evidencia-se o epitélio estratificado não ceratinizado. Figura 4. B: Aponta-se o infiltrado inflamatório crônico.
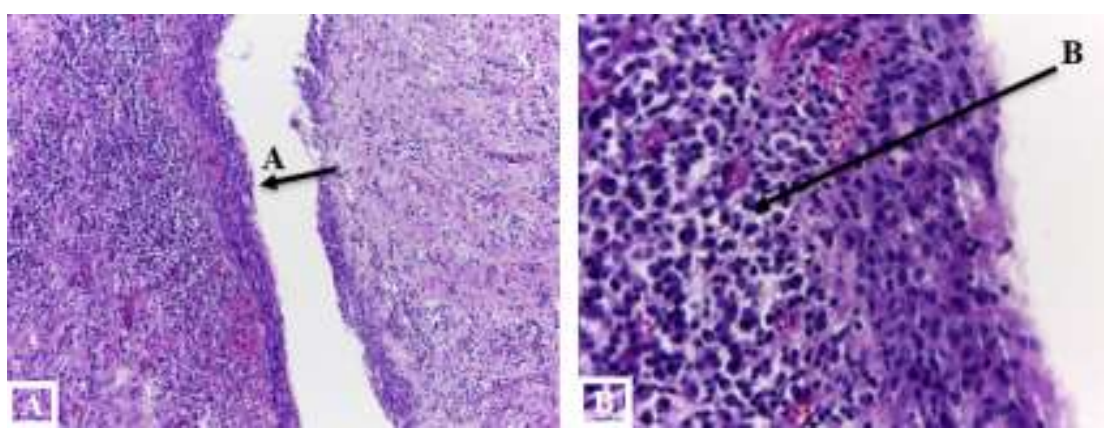

Fonte: Autores.

As características microscópicas evidenciaram um cisto revestido por epitélio escamoso estratificado não ceratinizado, além de conter áreas com infiltrado inflamatório crônico presente na cápsula de tecido conjuntivo fibroso circunjacente. Essas características são clássicas de um cisto inflamatório, portanto, não são suficientes se utilizadas isoladamente para o diagnóstico.

Foram realizadas consultas de acompanhamento com 15 dias e, posteriormente, com 30 dias para revisão do pósoperatório. Observando-se a ferida cirúrgica na região do 46 em boa cicatrização e o elemento 46 com coroa totalmente exposta (Figuras 5 e 6 ). 
Research, Society and Development, v. 10, n. 5, e19310515018, 2021

(CC BY 4.0) | ISSN 2525-3409 | DOI: http://dx.doi.org/10.33448/rsd-v10i5.15018

Figura 5. Acompanhamento após 15 dias.

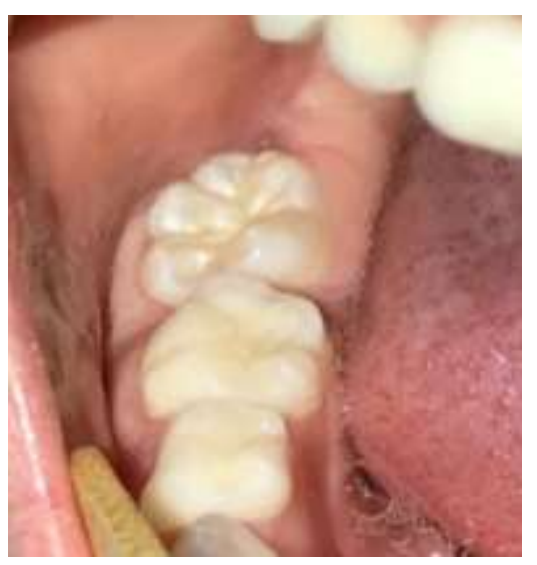

Fonte: Autores.

Figura 6: Acompanhamento após 30 dias.

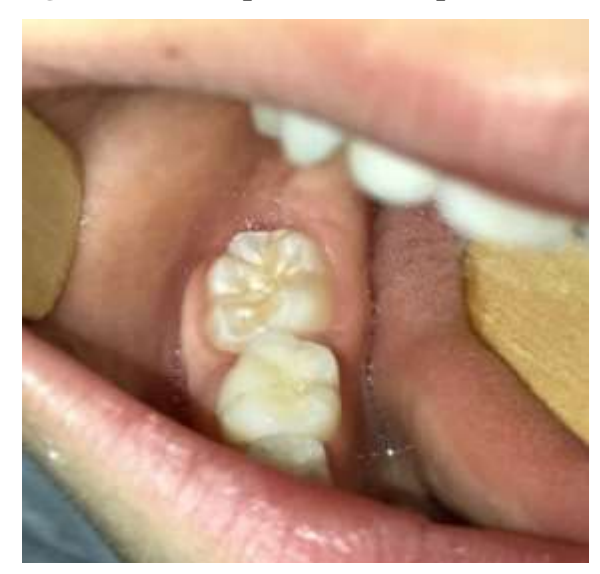

Fonte: Autores.

Após 90 dias foi realizado um exame de TCFC de controle pós-operatório. Observou-se regressão e desaparecimento da lesão cística, ausência de sinais de recidiva e boa consolidação óssea (Figuras 7A e 7B). 
Figura 7. Exame de TCFC de controle pós-operatório. (A) Reconstrução panorâmica e (B) Reconstrução 3D mostrando o elemento 46 erupcionado com rizogênese incompleta. (A) Reconstrução panorâmica, (B) Reconstrução 3D, (C) Cortes transaxiais e (E) Cortes Axiais evidenciando a reparação óssea óssea sugerindo o desaparecimento da lesão cística.

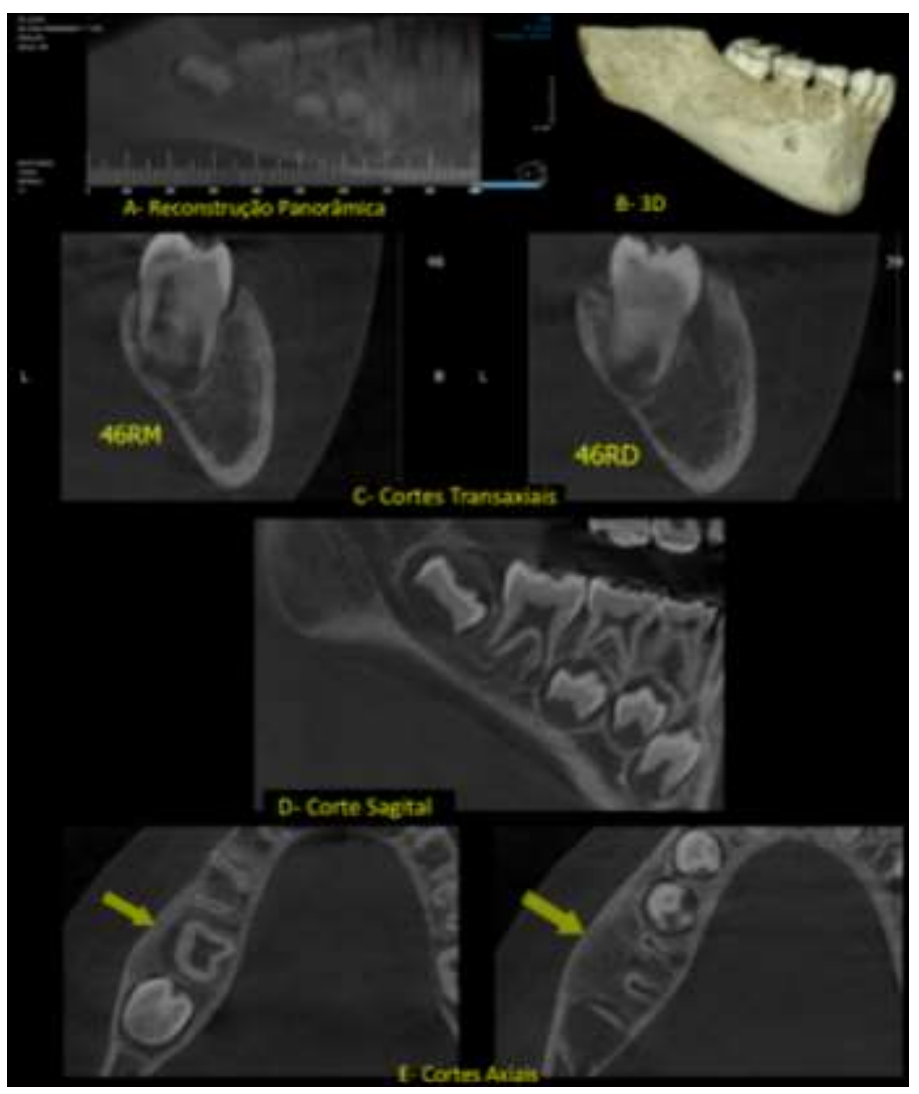

Fonte: Autores.

\section{Discussão}

Ao longo dos anos percebe-se uma grande variação na literatura de nomenclaturas usadas para o CBV (Friedrich, Scheuer, \& Zustin, 2014; Gomez, 2016; Lino et al., 2017; Pompura, Sandor, \& Stonemam, 1997; Shohat, Buchner, \& Taicher, 2003; Ramos et al., 2012). Esse fator, está diretamente relacionado aos relatos de baixa incidência dessa patologia (Bautista et al., 2019) e atualmente, o termo mais utilizado é Cisto da Bifurcação Vestibular (Grauwe et al., 2018; Oenning et al., 2018; Kim et al., 2018).

A etiopatogênese do CBV é alvo de grandes discussões. Algumas possibilidades têm sido apresentadas, como a de que sua origem seria no epitélio sulcular ou resultante da proliferação dos restos epiteliais de Malassez (Ramos et al., 2012; Shohat, Buchner, \& Taicher, 2003). A teoria de maior concordância entre pesquisadores é que esse cisto se desenvolva a partir do epitélio do órgão do esmalte, na época de erupção do elemento dentário envolvido (Friedrich, Scheuer, \& Zustin, 2014; Gomez, 2016; Grauwe et al., 2018; Lino et al., 2017; Oenning et al., 2018; Pompura, Sandor, \& Stonemam, 1997; Shohat, Buchner, \& Taicher, 2003; Ramos et al., 2012), corroborando com o caso apresentado neste estudo.

Em sua maioria, o CBV ocorre na mandíbula de pacientes pediátricos, mais especificamente no primeiro molar inferior permanente e menos frequente no segundo molar inferior permanente (Friedrich, Scheuer, \& Zustin, 2014). Concordando com o caso apresentado neste estudo, onde o paciente apresentava 7 anos de idade e foi afetado o primeiro molar permanente inferior, em processo eruptivo. Não há relatos encontrados na literatura da presença dessa patologia na maxila e nem o acometimento em indivíduos adultos (Oenning et al., 2018). 
Diversos autores afirmam que o CBV afeta a superfície vestibular de primeiros molares permanentes inferiores em erupção (Bautista et al., 2019; Friedrich, Scheuer, \& Zustin, 2014; Gomez, 2016; Grauwe et al., 2018; Lino et al., 2017; Oenning et al., 2018; Pompura, Sandor, \& Stonemam, 1997; Shohat, Buchner, \& Taicher, 2003; Ramos et al., 2012).

Os autores corroboraram que os dentes associados apresentaram uma alteração relacionada ao momento de erupção, com uma inclinação para vestibular da coroa e uma profunda bolsa periodontal, podendo haver algum inchaço ou sensibilidade, e geralmente, expansão óssea intraoral, sensível a palpação, de acordo com o grau de infecção (Bautista et al., 2019; Grauwe et al., 2018; Oenning et al., 2018; Pompura, Sandor, \& Stonemam, 1997; Ramos et al., 2012). Tais características assemelham-se com as encontradas neste relato de caso.

Diversos autores afirmaram que a patologia é acompanhada por aumento de volume doloroso (Bautista et al., 2019; Friedrich, Scheuer, \& Zustin, 2014; Gomez, 2016; Grauwe et al., 2018; Lino et al., 2017; Oenning et al., 2018; Pompura, Sandor, \& Stonemam, 1997; Shohat, Buchner, \& Taicher, 2003; Ramos et al., 2012), e essa mesma afirmação é encontrada neste relato. Entretanto, para Lino et al. (2017) a dor não é uma característica comum dessa patologia.

Além disso, é consenso na literatura que, apesar da proximidade com o canal mandibular, não há relatos de casos em que o paciente apresentasse parestesia relacionada a agressividade e expansão da lesão, ou por trauma no ato cirúrgico do nervo alveolar inferior (Bautista et al., 2019; Friedrich, Scheuer, \& Zustin, 2014; Gomez, 2016; Grauwe et al., 2018; Lino et al., 2017; Oenning et al., 2018; Pompura, Sandor, \& Stonemam, 1997; Shohat, Buchner, \& Taicher, 2003; Ramos et al., 2012; Kim et al., 2018). O paciente do presente relato também não apresentou parestesia, somando-se a literatura estudada.

Histologicamente, é revestido por células não queratinizadas, epitélio estratificado com uma parede de cisto fibroso apresentando inflamação crônica (Bautista et al., 2019; Gomez, 2016; Pompura, Sandor, \& Stonemam, 1997; Kim et al., 2018; Waldron, 2009). No entanto, o diagnóstico deste cisto não pode ser verificado apenas com as características histopatológicas, pois estas não são específicas (Gomez, 2016; Oenning et al., 2018). As características histológicas são comuns a todos cistos inflamatórios, que é um fator importante para enfatizar a importância do exame bidimensional e tridimensional de imagem para uma correta interpretação (Bautista et al., 2019; Gomez, 2016; Oenning et al., 2018; Kim et al., 2018). Consoando com a literatura, este caso demonstrou um revestimento clássico de cisto inflamatório, sendo o diagnóstico inconclusivo.

Sendo assim, ao analisar o aspecto microscópico, diversos estudos mostraram que o critério do histopatológico é duvidoso, e o mais aceitável e eficaz para diferenciação do CBV dos demais cistos inflamatórios é o teste de vitalidade, além de exames radiográficos (Grauwe et al., 2018; Kim et al., 2018; Oenning et al., 2018).

As características radiográficas são essenciais para auxiliar a reconhecer e distinguir as lesões císticas (Oenning et al., 2018). No exame bidimensional observa-se uma lesão radiolúcida, unilocular, bem-circunscrita (Ramos et al., 2012; Shohat, Buchner, \& Taicher, 2003; Waldron, 2009). Gomez (2016) acrescenta que a imagem radiolúcida é em forma de "'U", envolvendo todo o elemento. Pode-se atentar além dessas características, duas outras bastante peculiar, o espaço do ligamento periodontal e lâmina dura permanecem inalterados (Bautista et al., 2019; Gomez, 2016; Kim et al., 2018).

Com o auxílio do exame tridimensional, nota-se ainda, especialmente na vista axial e coronal uma expansão óssea no local, perda óssea na furca do dente, e a inclinação das raízes para cortical lingual, que é normalmente uma característica peculiar encontrada no CBV (Bautista et al., 2019; Kim et al., 2018; Waldron, 2009). O relato apresentado neste trabalho, está de acordo com as características bidimensionais e tridimensionais encontrados na literatura.

As entidades patológicas abordadas como diagnostico diferencial são: $\mathrm{O}$ cisto dentígero, radicular e o paradentário (Bautista et al., 2019; Gomez, 2016; Grauwe et al., 2018; Waldron, 2009). Dentre essas lesões, o cisto paradentário é o que mais se confunde com o cisto da bifurcação vestibular, especificamente em virtude da falta de conhecimento do CBV (Rodrigues, 2011), visto que, observa-se em estudos realizados, que as características clínicas e radiográficas são divergentes 
quando colocadas em comparação. Por isso, em razão do possível diagnóstico errôneo pode a incidência do CBV ser, talvez, maior do que algumas investigações mostram (Bautista et al., 2019).

A diferenciação da principal hipótese diagnóstica, o cisto paradentário, é bem abordada com base em (Gomez, 2016; Waldron, 2009), sendo afetado o terceiro molar semi-incluso na face distal, seguido por um histórico de pericoronarite e com maior predominância em pacientes adultos. Já o cisto abordado neste trabalho, encontra-se na região vestibular dos primeiros molares inferiores e tem predileção por crianças (Bautista et al., 2019; Friedrich, Scheuer, \& Zustin, 2014; Gomez, 2016; Grauwe et al., 2018; Lino et al., 2017; Oenning et al., 2018; Ramos et al., 2012, Waldron, 2009).

$\mathrm{Na}$ literatura, diferentes opções de tratamento são relatadas, a depender da extensão da lesão (Grauwe et al., 2018). O tratamento do CBV mudou significativamente com o passar dos anos (Bautista et al., 2019; Shohat, Buchner, \& Taicher, 2003). Inicialmente, foi tratado com enucleação e extração do dente envolvido (Stonemam \& Worth, 1983). Atualmente, o tratamento de escolha tem sido enucleação sem a extração do dente envolvido (Bautista et al., 2019; Ellis III, 2015; Grauwe et al., 2018; Oenning et al., 2018; Kim et al., 2018). Nota-se ainda que as complicações mais frequentes relacionadas a essa patologia é a perda do elemento dentário envolvido e parestesia do nervo alveolar inferior (Bautista et al., 2019; Oenning et al., 2018).

\section{Considerações Finais}

Concluiu-se que o caso relatado de cisto da bifurcação vestibular corrobora a literatura sendo uma doença pediátrica que ocorre na área de bifurcação da face vestibular de um primeiro molar inferior permanente em erupção e ocorrem principalmente na primeira década de vida. O diagnóstico é com base nas características clínicas, radiológicas e histológicas. Sendo as histológicas, insuficientes para um diagnóstico preciso. O tratamento mais preconizado atualmente, é a enucleação do cisto sem extração do dente associado. Este tratamento possui bons resultados a longo prazo de acordo com a literatura e sem recidivas.

O conhecimento das características distintas do CBV é importante para diagnóstico e tratamento, a fim de evitar-se condutas inadequadas e sequelas ao paciente. Ademais, sugere-se a realização de mais pesquisas para comprovar as diferenças existentes entre os tipos de cistos odontogênicos inflamatórios e as diversas formas de condutas para tratamento.

\section{Referências}

Bautista, C. R. G., Milhan, N. V. M., Ankha, M. D. V. E. A., Prado, R. F. D. P., Cavalcante, A. S. R., \& Lopes, S. L. P. C. (2019). Bilateral mandibular buccal bifurcation cyst: a case report emphasizing the role of imaging examination in the diagnosis. Autopsy and Case Reports, 9(2), 1-7.

Ellis III, E. (2015). Tratamento cirúrgico das lesões patológicas orais. In: Hupp, J. R., Ellis, E., \& Tucker, M. R. Cirurgia oral e maxilofacial contemporânea. Elsevier.

Faria, N. S. F., Monteiro, V. P., Barros, A. A. P., \& Nicolau, R. A. (2013). Técnica de marsupialização e enucleação para o tratamento de cistos de grandes proporções - Relato de caso. XVII Encontro Latino Americano de Iniciação Científica, XIII Encontro Latino Americano de Pós-Graduação e III Encontro de Iniciação à Docência.

Friedrich, R. E., Scheuer, H. A., \& Zustin, J. (2014). Inflammatory Paradental Cyst of the First Molar (Buccal Bifurcation Cyst) in a 6-Year-old Boy: Case Report with Respect to Immunohistochemical Findings. In Vivo, 28(3), 333-339.

Gomez, R. S. (2016). Cistos odontogênicos e não odontogênicos. In: Almeida, O. P., Loyola, A. M., Silva, A. R. S., Altemani, A. M., Andrade, B. A. B., \& Perez, D. E. C. Artes Médicas.

Graner, E., Jorge, J. J., Almeida, O. P., Vargas, P. A., \& Colleta, R. D. (2015). Cistos Odontogênicos. https://w2.fop.unicamp.br/ddo/patologia/downloads/db301_un3_Aula29-30Cistos_desenvolvimento.pdf. Acesso em: 26 mai. 2020.

Grauwe, A., Mangione, F., Mitsea, A., Kalyvas, D., Yfanti, Z., \& Ahbab, G. (2018). Update on a rare mandibular osteolytic lesion in childhood: the buccal bifurcation cyst. Brazilian Journalism Research, 4(2), 2-6.

Kim, H. R., Nam, S. H., Kim, H. J., \& Choi, S. Y. (2018). Buccal Bifurcation Cyst: Two Case Reports and a Literature Review. The Journal of Clinical Pediatric Dentistry, 42(3), 221-224. 
Research, Society and Development, v. 10, n. 5, e19310515018, 2021

(CC BY 4.0) | ISSN 2525-3409 | DOI: http://dx.doi.org/10.33448/rsd-v10i5.15018

Lino, F. A.; Pereira, M. C.; Doses, P. L. A.; Pereira, A. A. C.; Goulart, M. C. V., \& Miranda, R. T. (2017). Cisto Inflamatório da Bifurcação Vestibular: Relato de Caso Clínico. Revista Intercâmbio, 12(29), 1-3.

Oenning, A. C., Oliveira, L. B., Junqueira, J. L. C., \& Sousa-melo, S. L. (2018). Buccal bifurcation cyst as an incidental finding in cone beam computed tomography scans. $R G O, 66(4), 38-59$.

Pereira, A. S., Shitsuka, D. M., Parreira, F. J. \& Shitsuka, R. (2018). Metodologia da pesquisa científica. UFSM. https://repositorio.ufsm.br/bitstream/handl e/1/158 24/LicComputacao_MetodologiaPesquisaCientifica.pdf?sequence=1

Pompura, J. R., Sándor, G. K. B., \& Stoneman, D. W. (1997). The buccal bifurcation cyst: A prospective study of treatment outcomes in 44 sites. Oral Surgery, Oral Medicine, Oral Pathology, Oral Radiology, and Endodontics, 83(2), 215-221.

Ramos, L. M. A., Vargas, P. A., Colleta, R. D., Almeida, O. P., \& Lopes M. A. (2012). Bilateral Buccal Bifurcation Cyst: Case Report and Literature Review. Head and Neck Pathology, 6(4), 455-459.

Rodrigues, A. P. M. B. S. (2011). Cistos odontogênicos inflamatórios: revisão de literatura. Trabalho de Conclusão de Curso (Graduação em Odontologia), Pontifícia Universidade Católica do Rio Grande do Sul, 34.

Santos, L. C. S., Ramos, E. A. G., Meira, T. M., Figueiredo, C. R. L. V., \& Santos, J. N. S. (2006). Etiopatogenia do cisto radicular. Parte I. Revista de ciências médicas biológicas, 5(2), 69-74.

Shohat, I., Buchner, A., \& Taicher, S. (2003). Mandibular buccal bifurcation cyst: Enucleation without extraction. International Journal of Oral and Maxillofacial Surgery, 32(6), 610-3.

Stoneman, D. W., \& Worth, H. M. (1983). The mandibular infected buccal cyst. Dent Radiogr Photogr, 56(1), 1-14

Tolentino, E. S. (2018). Nova classificação de tumores odontogênicos: o que mudou? Revista da Faculdade de Odontologia, 23(1), 119-123.

Waldron, C. A. (2009). Cistos e tumores odontogênicos. In: Neville, B. W.; Damm, D. D., Allen, C. M., \& Bouquot, J. E., Patologia oral e maxilofacial. Elsevier. 